the first 16 numbers generated by this process. An asterisk marks each integer whose digits represent a required permutation. The other integers were rejected because of the occurrence of repeated digits.

$\begin{array}{clcc}\text { Sequence } & \text { Integer } & \text { Sequence } & \text { Integer } \\ 1 & 0123^{*} & 9 & 0303 \\ 2 & 0132^{*} & 10 & 0312^{*} \\ 3 & 0201 & 11 & 0321^{*} \\ 4 & 0210 & 12 & 0330 \\ 5 & 0213^{*} & 13 & 0333 \\ 6 & 0222 & 14 & 1002 \\ 7 & 0231^{*} & 15 & 1011 \\ 8 & 0300 & 16 & 1020\end{array}$

4. Adaptation to a Computer. In a computer such as the IBM 7090 where convert instructions are available it is easy to do radix $k$ arithmetic. Otherwise one could simulate the process by adding 9 digit-wise and testing the resulting sum for having unique digits each one of which is one of the original $k$ digits.

5. Acknowledgments. This method was developed when the author was with the Statistics Department, Agricultural Experiment Station, University of Florida, Gainesville, Florida, in connection with the problem of obtaining a particular arrangement of the rows of a Latin square. He wishes to thank Mark Robinson of Martin Marietta Corp. for suggestions concerning the writing of the manuscript.

Computer Applications Department

Martin Marietta Corporation

Orlando, Florida

1. C. B. Tomprins, "Machine attacks on problems whose variables are permutations," Proceedings of Symposia in Applied Mathematics, v. VI, Numerical Analysis, McGraw-Hill, New York, 1956, p. 195-211.

2. D. H. LEHMER, "Teaching combinatorial tricks to a computer," Proceedings of Symposia in Applied Mathematics, v. X, Combinatorial Analysis, American Mathematical Society, Providence, R. I., 1960, p. 179-193.

3. MARK B. Wells, "Generation of permutations by transposition," Math. Comp. v. 15, 1961, p. 192-195.

\title{
Multiple Quadrature with Central Differences on One Line
}

\section{By Herbert E. Salzer}

Abstract. The coefficients $A_{2 m}^{n}$ in the $n$-fold quadrature formulas for the stepwise integration of $(1) y^{(n)}=f\left(x, y, y^{\prime}, \cdots, y^{(n-1)}\right)$, at intervals of $h$, namely, for $n$ even, (2) $\delta^{n} y_{0}=h^{n} \sum_{m=1}^{10}\left(1+A_{2 m}^{n} \delta^{2 m}\right) f_{0}+\cdots$, for $n$ odd, (3) $\mu \delta^{n} y_{0}=h^{n} \sum_{m=1}^{10}$ $\left(1+A_{2 m}^{n} \delta^{2 m}\right) f_{0}+\cdots$, are tabulated exactly for $n=1(1) 6, m=1(1) 10$. They were calculated from the well-known symbolic formulas (4) $\delta^{n} y=(\delta / D)^{n} f,(5)$ $(\delta / D)^{n}=\left(\delta h / 2 \sinh ^{-1}(\delta / 2)\right)^{n}$ and $(6) \mu=\left(1+\delta^{2} / 4\right)^{1 / 2}=1+\frac{\delta^{2}}{8}-\frac{\delta^{4}}{128}+\frac{\delta^{6}}{1024}-$ 
$\frac{5 \delta^{8}}{32768}+\cdots$. For calculating $y^{(r)}$, replace $n$ by $n-r$ in (2) and (3). Use of

and (3) avoids the solution of (1) by simultaneous lower-order systems for $n>1$, as well as mid-interval tabular arguments, requires only even-order differences, on a single line, and provides great accuracy due to rapid decrease of $A_{2 m}^{n}$ as $m$ increases. However, the integration may be slowed down by the need to estimate and refine iteratively the later values of $y, y^{\prime}, \cdots, y^{(n-1)}$ required in $\delta^{2 m} f_{0}$. Reference to earlier collected formulas of Legendre, Oppolzer, Thiele, Lindow, Salzer, Milne and Buckingham, reveals that Thiele and Buckingham come closest to (2), (3), as their works contain schemes that involve just tabular arguments throughout. For $n$ odd, they give formulas that are based upon the series in $\delta^{2 m}$ for $(1 / \mu)(\delta / D)^{n}$ instead of $\mu(\delta / D)^{n}$ as in the present arrangement.

\section{Purpose and Scope of Tabulated Formulas. Given a differential equation}

$$
y^{(n)}=f\left(x, y, y^{\prime}, \cdots, y^{(n-1)}\right),
$$

and a sufficient number of starting values at intervals of $h$, there are very convenient numerical integration formulas for obtaining either $\delta^{n} y_{0}$, for $n$ even, or $\mu \delta^{n} y_{0}$, for $n$ odd, in terms of just the even-order central differences of $f \equiv f\left(x, y, y^{\prime}, \cdots, y^{(n-1)}\right)$ at $x=x_{0}$, denoted by $\delta^{2 m} f_{0}$. This article tabulates the exact values of $A_{2 m}^{n}$, the coefficients of $\delta^{2 m} f_{0}$, for $n=1(1) 6, m=1(1) 10$, in the following numerical integration formulas:

$$
\begin{aligned}
& \delta^{n} y_{0}=h^{n} \sum_{m=1}^{10}\left(1+A_{2 m}^{n} \delta^{2 m}\right) f_{0}+\cdots, \quad \text { for } n \text { even, and } \\
& \mu \delta^{n} y_{0}=h^{n} \sum_{m=1}^{10}\left(1+A_{2 m}^{n} \delta^{2 m}\right) f_{0}+\cdots, \quad \text { for } n \text { odd } .
\end{aligned}
$$

The computation of $A_{2 m}^{n}$ was based upon the symbolic form of (1), or $D^{n} y=f$, from which

$$
\delta^{n} y=(\delta / D)^{n} f
$$

The well-known operational formula,

$$
(\delta / D)^{n}=\left(\delta h / 2 \sinh ^{-1}(\delta / 2)\right)^{n},
$$

was used to obtain the coefficients of $\delta^{2 m}$ in the series for $(\delta / D)^{n}$. For even $n$, this yielded (2). For odd $n,(5)$ produces integration formulas that express mid-interval values of $y$ in terms of tabular values of $f$. To obtain (3), which involves tabular values of both $y$ and $f$, we multiply (5) by $\mu$, giving $\mu$, on the left side, the numerical interpretation of a mean central operator $\frac{1}{2}\left(E^{1 / 2}+E^{-1 / 2}\right)$, and considering $\mu$, on the right side, a symbolic even function of $\delta$ according to

$$
\mu=\left(1+\delta^{2} / 4\right)^{1 / 2}=1+\frac{\delta^{2}}{8}-\frac{\delta^{4}}{128}+\frac{\delta^{6}}{1024}-\frac{5 \delta^{8}}{32768}+\cdots
$$

Integration of (1) also requires formulas for the stepwise determination of the derivatives $y^{(r)}, r=1(1) n-1$. By noting that $D^{n-r} y^{(r)}=f$, we can still employ (2) and (3), as well as the same quantities $\delta^{2 m} f_{0}$, merely replacing $n$ by $n-r$. 
In the use of (2) and (3) we avoid the widespread practice of breaking up a higher-order equation into a simultaneous first-order system where each equation requires its own set of differences. Also there is no occurrence of formulas involving mid-interval arguments. Among the attractive features of this scheme is the employment of just alternate even-order differences that are on a single line. Besides the concise and economical appearance of (2), (3), the rapid rate of decrease of $A_{2 m}^{n}$ with increasing $m$ is seen to provide high accuracy.

On the dampening side, the user is reminded that the higher-order central differences of $f\left(x, y, y^{\prime}, \cdots, y^{(n-1)}\right)$ in (2) and (3) involve later values of $y, y^{\prime}, \cdots, y^{(n-1)}$ that must be estimated at first, probably by some kind of extrapolation. Then (2) and (3) might be used in some iterative refining scheme, the details depending upon the particular functional form of $f\left(x, y, y^{\prime}, \cdots, y^{(n-1)}\right)$, the nature of the problem, and the desired accuracy (all of which is a vast subject in itself).

2. Comparison with Earlier Work. The chief novelty in the present arrangement is the systematic use of the $\mu$-series in terms of $\delta^{2 m}$ to obtain (3) for any odd $n$ (see also Milne below). Two other authors (Thiele, Buckingham), by employing the series for $1 / \mu$ in terms of $\delta^{2 m}$, give formulas for odd $n$ that are closely related to (3), requiring just tabular arguments and avoiding the introduction of mid-interval arguments (as is done by Legendre, Oppolzer, Lindow). Presented chronologically, there is the following earlier work.

Legendre [1] gives the symbolic formula for the $(\delta / D)^{n}$ series in $\delta^{2 m}$ and the first few coefficients up to $n=6$.

Oppolzer [2] gives the exact coefficients for $(\delta / D)$ and $(\delta / D)^{2}$ up to $\delta^{20}$. His $(\delta / D)$ coefficients checked with those in Salzer [5]. His $(\delta / D)^{2}$ coefficients checked with $A_{2 m}^{2}$ here, except for his coefficient $Q_{2}{ }^{14}\left(=A_{16}^{2}\right)$ not in lowest terms by a factor of 9 .

Thiele [3] gives the exact values of the first five coefficients for $D^{-n}$ and $(1 / \mu) D^{-n}$, which is the same as $(\delta / D)^{n}$ in terms of $\delta^{2 m}$ and $\mu \delta^{2 m}$ up to $m=5$, for $n=1(1) 5$.

Lindow [4], who gives some central difference formulas up to triple quadrature, also gives the exact values of $A_{2 m}^{2}$, for $m=1(1) 7$. $\delta^{50}$.

Salzer [5] tabulates the coefficients of $\delta / D$, exactly through $\delta^{20}$, then $18 \mathrm{D}$ through

Milne [6] happens to give $2 A_{2 m}^{1}, m=1(1) 5$, in the first of a series of formulas for $\int_{x_{0}-r h}^{x_{0}+r h} f(x) d x, r=1(1) 5$, in terms of $\delta^{2 m} f_{0}$.

Salzer [7] gives the coefficients of $\delta_{0}{ }^{2 m}$ and $\delta_{1}{ }^{2 m}$ obtained by $k$-fold quadrature of Everett's formula; for $k=2$, exactly up to $m=10$, then $16 \mathrm{D}$ up to $m=24$; for $k=3(1) 6$, exactly for $m=0$ and $8 \mathrm{~S}$ for $m=1(1) 10$. These differ from the other coefficients in that they occupy two lines for central differences instead of one. They are mentioned here because of their similar purpose and the large extent to which they have been tabulated.

Buckingham [8] gives the coefficients of $(\delta / D)^{n}$ and $(1 / \mu)(\delta / D)^{n}, n=1(1) 4$, through $\delta^{8}$. As in Thiele [3], this includes an integration scheme involving just tabular arguments for every $n$. Thus, by expressing $(\delta / D)^{n}$ for odd $n$ as $\left\{(1 / \mu)(\delta / D)^{n}\right\} \mu$, and choosing $x_{0}+h / 2$ for the argument, Buckingham obtains odd-order central differences of the integral, at mid-intervals, in terms of mean central even-order 
differences, also at mid-intervals, so that both members involve $y$ and $f$ for just tabular arguments. However, it appears to the author that for $n$ odd there is less total computation involved in using (3) for $\mu(\delta / D)^{n}$, where the slight extra work of finding $\mu \delta^{n} y_{0}$ instead of $\delta^{n} y_{1 / 2}$ is more than compensated for by not having to average all the quantities $\delta^{2 m} f_{0}$ and $\delta^{2 m} f_{1}$, as is done in the Buckingham-Thiele procedure which uses $(1 / \mu)(\delta / D)^{n}$ with the mean central differences $\mu \delta^{2 m} f_{1 / 2}$.

3. Integration Formulas for $y^{(n)}=f\left(x, y, y^{\prime}, \cdots, y^{(n-1)}\right)$

$$
\begin{aligned}
n=1: \mu \delta y_{0}= & h\left(1+\frac{\delta^{2}}{6}-\frac{\delta^{4}}{180}+\frac{\delta^{6}}{1512}-\frac{23}{226800} \delta^{8}+\frac{263}{14968800} \delta^{10}\right. \\
& -\frac{133787}{40864824000} \delta^{12}+\frac{157009}{245188944000} \delta^{14} \\
& -\frac{16215071}{125046361440000} \delta^{16}+\frac{2689453969}{99786996429120000} \delta^{18} \\
& \left.-\frac{26893118531}{4704244117372800000} \delta^{20}\right) f_{0}
\end{aligned}
$$

$n=2: \quad \delta^{2} y_{0}=h^{2}\left(1+\frac{\delta^{2}}{12}-\frac{\delta^{4}}{240}+\frac{31}{60480} \delta^{6}-\frac{289}{3628800} \delta^{8}+\frac{317}{22809600} \delta^{10}\right.$

$-\frac{6803477}{2615348736000} \delta^{12}+\frac{3203699}{6276836966400} \delta^{14}$

$-\frac{73691749}{711374856192000} \delta^{16}+\frac{220387795651}{10218188434341888000} \delta^{18}$

$\left.-\frac{154473473256043}{33720021833328230400000} \delta^{20}\right) f_{0}$

$n=3: \quad \mu \delta^{3} y_{0}=h^{3}\left(1+\frac{\delta^{2}}{t}+\frac{\delta^{4}}{240}+\frac{\delta^{6}}{60480}-\frac{\delta^{8}}{57600}+\frac{661}{159667200} \delta^{10}\right.$

$-\frac{ \pm 65967}{523069747200} \delta^{12}+\frac{396079}{2092278988800} \delta^{14}$

$-\frac{9595529}{237124952064000} \delta^{16}+\frac{17857425881}{2043637686868377600} \delta^{18}$

$\left.-\frac{21432730664071}{11240007277776076800000} \delta^{20}\right) f_{0}$

$n=4: \quad \delta^{4} y_{0}=h^{4}\left(1+\frac{\delta^{2}}{6}-\frac{\delta^{4}}{720}+\frac{\delta^{6}}{3024}-\frac{41}{725760} \delta^{8}+\frac{491}{47900160} \delta^{10}\right.$

$-\frac{3+1749}{17+356582400} \delta^{12}+\frac{50971}{130767436800} \delta^{14}$

$-\frac{170+03199}{2134124568576000} \delta^{16}+\frac{85513758923}{5109094217170944000} \delta^{18}$ 


$$
\begin{aligned}
n=5: \quad \mu \delta^{5} y_{0}=h^{5} & \left.-\frac{17213818448999}{4817145976189747200000} \delta^{20}\right) f_{0} \\
& +\frac{13283}{174356582400} \delta^{12}+\frac{\delta^{2}}{1046139494400} \delta^{14} \\
& -\frac{966067}{237124952064000} \delta^{16}+\frac{475770541}{364935301226496000} \delta^{8}-\frac{11}{7904} \delta^{10} \\
& \left.-\frac{241939616497}{688163710884249600000} \delta^{20}\right) f_{0} \\
n=6: \quad \delta^{6} y_{0}=h^{6} & \left(1+\frac{\delta^{2}}{4}+\frac{\delta^{4}}{120}+\frac{\delta^{6}}{30240}-\frac{\delta^{8}}{57600}+\frac{31}{7603200} \delta^{10}\right. \\
& -\frac{27257}{31135104000} \delta^{12}+\frac{11581}{62270208000} \delta^{14} \\
& -\frac{1554079}{39086530560000} \delta^{16}+\frac{12535354591}{1459741204905984000} \delta^{18} \\
& \left.-\frac{1504839712643}{802857662698291200000} \delta^{20}\right) f_{0}
\end{aligned}
$$

General Dynamics/Astronautics

San Diego, California

1. A. M. Legendre, Traité des Fonctions Elliptiques, v. 2, Paris, 1826, Chapter 3, p. 41-60 (For errors, see $M T A C$, v. 5, 1951, p. 27).

2. T. R. OpPolzer, Lehrbuch zur Bahnbestimmung der Cometen und Planeten, v. 2, W. Engelmann, Leipzig, 1880, p. 35, 53-54, 545, 596.

3. T. N. ThIELe, Interpolationsrechnung, B. G. Teubner, Leipzig, 1909, p. 95-97. (Some misprints are noted in Math. Comp., v. 15, 1961, p. 321.)

4. M. Lindow, Numerische Infinitesimalrechnung, F. Dümmler, Berlin and Bonn, 1928, p. 170-171.

5. H. E. SALzER, "Coefficients for mid-interval numerical integration with central differences," Phil. Mag., ser. 7, v. 36, 1945, p. 216-218.

6. W. E. Milne, Numerical Calculus, Princeton, 1949, p. 196-197.

7. H. E. SALZER, "Coefficients for repeated integration with central differences," J. Math. Phys., v. 28, 1949, p. 54-61.

8. R. A. Buckingham, Numerical Methods, Pitman Publishing Corp., New York and London, 1957. p. 150-154. (For errors, see Math. Comp., v. 15, 1961, p. 319.). 